\title{
Dwarf Galaxies with Optical Signatures of Accreting Massive Black Holes
}

\author{
Amy Reines, J. Greene and M. Geha \\ National Radio Astronomy Observatory (NRAO, USA \\ E-mail: areines@nrao.edu
}

\begin{abstract}
Supermassive black holes (BHs) live at the heart of essentially all massive galaxies with bulges, power AGN, and are thought to be important agents in the evolution of their hosts. Observations of high-redshift quasars demonstrate that supermassive BHs must start out with masses considerably in excess of normal stellar-mass BHs. However, we do not know how the initial "seed" BHs formed in the early Universe, how massive they were originally, or what types of galaxies they formed in. While direct observations of distant seed BHs and their hosts in the infant Universe are unobtainable with current capabilities, models of $\mathrm{BH}$ growth in a cosmological context indicate that present-day dwarf galaxies can place valuable constraints on seed masses and distinguish between various seed formation mechanisms at early times. Using optical spectroscopy from the SDSS, we have systematically assembled the largest sample of dwarf galaxies hosting AGN to date. These dwarf galaxies have stellar masses comparable to the Magellanic Clouds and contain some of the least-massive supermassive BHs known. I will present results from this study and discuss our ongoing efforts to find additional examples of AGN in dwarfs and help constrain theories for the formation of the first seed BHs at high redshift.
\end{abstract}

